\title{
THE BETEL QUID CHEWING HABIT: WHY IT SHOULD BE STOPPED AND WHAT CAN BE DONE TO STOP THIS HABIT?
}

R. Saub. The Betel Quid Chewing Habit: Why it should be stopped and what can be done to stop this habit? Annal Dent Univ Malaya 2001; 8: 62-66.

\begin{abstract}
The habit of chewing betel quid has been practised since ancient times. Although the world has gone through modernization, a significant proportion of people still practices this habit. Substantial evidence has shown that betel quid chewing is associated with the occurrence of oral cancer and precancerous lesions, which has a tremendous psychosocial impact on an individual's life. Thus it becomes significantly important to dentistry to look into this matter. Since betel quid chewing is one of the causes of oral cancer, effort in changing this habit is essential. This article addresses this issue.
\end{abstract}

Key words: betel quid chewing, Protection-Motivation model, oral cancer.

\section{INTRODUCTION}

Archeological evidence suggests that betel quid chewing has been practised since ancient times and has survived into the twentieth century (1). The habit can be described as the practice of masticating a quid of ingredients, including the seed of areca nut (betel nut), the leaf of vine piper betie, and lime (2). It was estimated that between $10-20 \%$ of the world population chew betel quid (3). This habit is commonly practised in Southeast Asia, the Indian subcontinent, the Western Pacific and other peripheral areas and it is totally alien to the Western world (1). However, people who migrated from this area to Western countries such as North America brought along this custom (2-4).

Historically, the habit is practised across class, sex, or age (1). However, this situation has changed, where in most parts of the Southeast Asia, the habit of betel quid chewing is slowly disappearing and is practised only among the older generation. However, in other countries such as in Taiwan, an increased number of betel quid chewers is found among the adolescence (5). It is also observed that in India this habit has been picked up by the younger generation due to the commercial product pan masala (containing areca nut) and gutka (containing areca nut and tobacco) (6). These products have been heavily advertised, intensively promoted and widely marketed all over India. In Malaysia the habit of betel quid chewing is commonly found among certain ethnic groups, namely the indigenous people of Sabah and Sarawak and the Indians who are usually from a low socioeconomic group (7). Unlike smoking, betel quid chewing is regarded as normal practice among women in most of the eastern culture. However, it is anticipated
Article of Interest

R. Saub
Department of Community Dentistry
Faculty of Dentistry
University Malaya
50603 Kuala Lumpur
MALAYSIA

that with increasing urbanisation and education, this habit is becoming somewhat unacceptable.

The essential ingredients for betel quid chewing are areca nut also known as betel nut, a leaf of the betelpepper and lime. However, other components may be added depending on the local customs and individual preference (1). The preparation for betel quid also varies from country to country. For example in Taiwan they usually chew betel nut with a lengthwise piece of unripe fruit with betel piper leaf and lime paste between the two halves of the nut (5). Chinese traditional herbs may be added but tobacco leaves are excluded. However, in Malaysia and the Indian subcontinent, the leaf is first daubed with lime paste and topped with thin slices of nut, then it is folded or rolled into a bite-size quid and chewed (1).

There are numerous studies that reported betel quid chewing being associated with oral cancer. Sankaranarayanan (1990) (8) in his review of oral cancer stated that this lesion is common where quid chewing, bidi smoking, alcohol and tobacco consumption are high. More than 100,000 new cases are reported every year in Southeast Asia. Oral cancer has been shown to have a great impact on the quality of life (9). Hence it is important to prevent this disease from occurring. Since betel quid chewing is one of the causes of oral cancer, effort on changing this habit is essential. This article addresses this issue.

Why do people chew betel quid?

There are several reasons why people chew betel quid. Rooney (1993) (1) in his book entitled "Betel chewing traditions in South-East Asia" stated that "the main reason for chewing betel seems to lie in the social affability produced by sharing a quid with friends'. Offering a quid to someone is a mark of hospitality. "When they received passing guests they entertain them not with tea but only with areca nut" observed the Chinese Ma Huan on a naval expedition to Southern Ocean, in the $15^{\text {th }}$ century. In some parts of Taiwan, for 
example, offering areca nut or a cigarette to others during a social gathering has become part of their custom (5).

Chewing betel quid is believed to have many health benefits. Pickwell et al (1994) (2) interviewed ten Cambodian women refugees in San Diego who chewed betel quid and observed that all of them had stained teeth. However, none of them perceived it as a problem and in fact three of them believed that chewing the quid strengthened their teeth. Three of the women made a connection between chewing after meals and better digestion of food. Lu et al. (1993) conducted a study to investigate the prevalence and characteristics of areca nut chewers among junior high school students (5). They found that almost half of the users stated that they chewed areca nut to dispel chills and refresh themselves. In India, betel quid is chewed for a number of reasons. Among them are to relieve toothache and indigestion, improve appetite, colour the mucosa, and overcome the boredom of life (10). According to a sixth-century Indian text, betel quid chewing is one of the eight "bhogas" or cardinal pleasures of life along with unguents, incense, women, garments, music, beds, food, and flowers and betel quid is an integral part of the Indian culture (1).

Among the Cambodians, stained teeth is a sign of maturity and aging (2). Therefore they chew betel quid to colour their teeth and lips. For the Cambodian "betel chewing is understood to be a rite passage from girlhood to womanhood. Even those women who experimented with the quid while very young did not habituate until their late teens or twenties. The defining landmark for them was the first birth of their child." (2).

\section{Betel quid chewing and oral cancer - the evidence}

Moore et al (2000) in their review on the global incidence of oral cancer using a cancer map (where data were compiled from the lateșt edition of cancer incidence in five continents and recent studies from various locations around the world), noted that there was a significant geographic variation in the incidence of oral cancer. The Indian subcontinent and parts of Asia reported high rates of oral cancer (11). For example, oral cancer accounts for approximately $3 \%$ of all oral cancers in the western world (12) whereas in India oral cancer represents $15 \%$ to $20 \%$ of all malignancies (8). This geographical variation may indicate the influence of the environment and culture on the occurrence of this disease. Moore et al (2000) concluded that oral cancer remain a serious health problem in many parts of the world with many regions reporting increasing incidence rates particularly in males (11). However, they noted that there were a paucity of reliable population based data from the developing countries.

Several epidemiological studies have shown an association between the habit of betel quid chewing and oral cancer and various precancerous lesions. The association has been consistent across many countries such as in India, Philippines, Malaysia, Bangladesh, Cambodia, and Thailand. Although most of the studies reported that chewing betel quid was associated with oral cancer, caution must be taken when interpreting these findings. This is because most studies did not clearly describe the chewing habits of subjects. The variation in the ingredients, preparation, and ways of chewing (frequency, duration) may have an impact on the findings. Thus a workshop was held in Kuala Lumpur, Malaysia in 1996 as an effort to bring some uniformity in the reporting of betel quid and tobacco chewing habits and associated oral mucosal lesions. As a result, some consensus was obtained (13).

Hirayama (1966), in an extensive study of oral and pharyngeal cancer in Southeast Asia, concluded that the chewing of tobacco and lime mixture plays an important role in the aetiology of oral cancer by causing cancer at the place where the quid is habitually kept (14). He also found that chewers have four to fifteen times greater risk than no-chewers to develop oral cancer. The probability of developing oral cancer is directly correlated with the duration and intensity of chewing.

Betel quid chewing was found to be associated with the occurrence of oral precancerous lesions, which are potentially malignant. In a cohort study by Gupta et al (1989) the relative risk of malignant transformation of oral submucous fibrosis compared to individuals without any precancerous lesions was reported as 397.3 after controlling for the use of tobacco (15). A cross-sectional population study conducted by Zain et al (1997) in Malaysia found that the Indians and indigenous people of Sabah and Sarawak have the highest prevalence of precancerous lesions and these two groups of the population were more likely to chew betel quid (16). Based on this study, they recommended that these two ethnic groups (Indian and Indigenous) should be considered as target groups in oral health promotion and prevention programmes. In another study by Ali et al (1997), a localized cross-sectional house to house survey of an elderly population in Malaysia reported that $72 \%$ of the Indian subjects had oral precancerous lesions (17). They also observed that among subjects with oral precancerous lesions, $4.2 \%$ were smokers, $12.7 \%$ were betel quid chewers, and $11.8 \%$ practised both. They suggested that the occurrence of oral precancerous lesion among the Malaysian elderly to be related to betel quid chewing. However, it must be noted that this study did not control for confounding factors in the analysis.

Animal studies have shown that the betel quid ingredients have carcinogenic properties. For example, a study on hamsters found that when the aqueous extracts or implantation of wax pellets containing powdered betel quid with or without tobacco was painted on the cheek pouch of stomach mucosa, forestomach carcinomas occurred (18).

Therefore it can be summarised that there are substantial evidences to suggest that betel quid chewing with or without tobacco is associated with oral cancer as well as initiating mucosal change, which has a greater chance to develop a malignancy.

\section{How to change this habit?}

Substantial evidences have shown that betel quid chewing is associated with the occurrence of oral cancer 
and precancer, which has a tremendous psychosocial impact on an individual's life. Thus it becomes significantly important to look into this matter. The question is how can we, as dental professionals help them (betel quid chewers) to stop from practicing this habit? Changing people's behaviour is not an easy task but efforts must be made to ensure that at least they are aware of the risk of their habit to their life and these efforts ultimately will lead to a change in their behaviour.

There are three approaches to healt 1 behaviour modification: attitudinal approaches, cognitivebehavioural approaches, and social engineering (19). Attitudinal approaches assume that if people can be persuaded that their current health habits are poor, they will be motivated to change those health habits. These approaches are good in predicting when people will be motivated to change their health behaviour. However, they do not really indicate which individuals modify their health behaviours successfully and which do not. The second approach is cognitive-behavioural approaches, which stress the fact that the behaviour, the environment in which it is embedded and the possession of necessary skills of behaviour change and maintenance, whether or not health behaviour will be practised or changed. The third approach is social engineering, which involves modifying the environment in ways that affect an individual's ability to practise particular health behaviour. Therefore in most cases all three approaches need to be utilized in order to ensure behaviour change in the individual or community.

An important step in the modification of health behaviour is to motivate an individual to want to change the behaviour. Researchers have found that the health behaviour can be changed by changing health beliefs, which ultimately changes health behaviour. Rogers in 1984 developed a model known as "Protection Motivation model" to understand health behaviour (19). This model maintains that the motivation to protect one's self from health threat is based on four beliefs: the threat is severe (magnitude of threat), one is personally vulnerable to the threat (i.e, its occurrence is likely), one is able to perform the response needed to reduce the threat (self-efficacy), and the response will be effective in overcoming the threat (response efficacy).

According to this model, when beliefs about these four factors are strong, then protection motivation is aroused, and an individual is more likely to change his or her attitudes toward health behaviour. The most important aspect of this model is that a direct communication toward changing attitudes about the severity of the threat, personal vulnerability, selfefficacy, and response efficacy. Therefore before this model can be applied, one needs to identify an individual's attitudes on these four variables and determine which if any need to be changed.

For the purpose of understanding the habit of betel quid chewing, this model (Protection Motivation model) was used. All four factors mentioned in the model have been identified by compiling data from several studies and the summary is shown in Figure 1. While there were numerous studies reported on smoking, very few studies were found in the literature that examined betel quid chewing habits. There was one qualitative study, which examined the knowledge about the health risk associated with betel quid chewing by interviewing 10 Cambodian

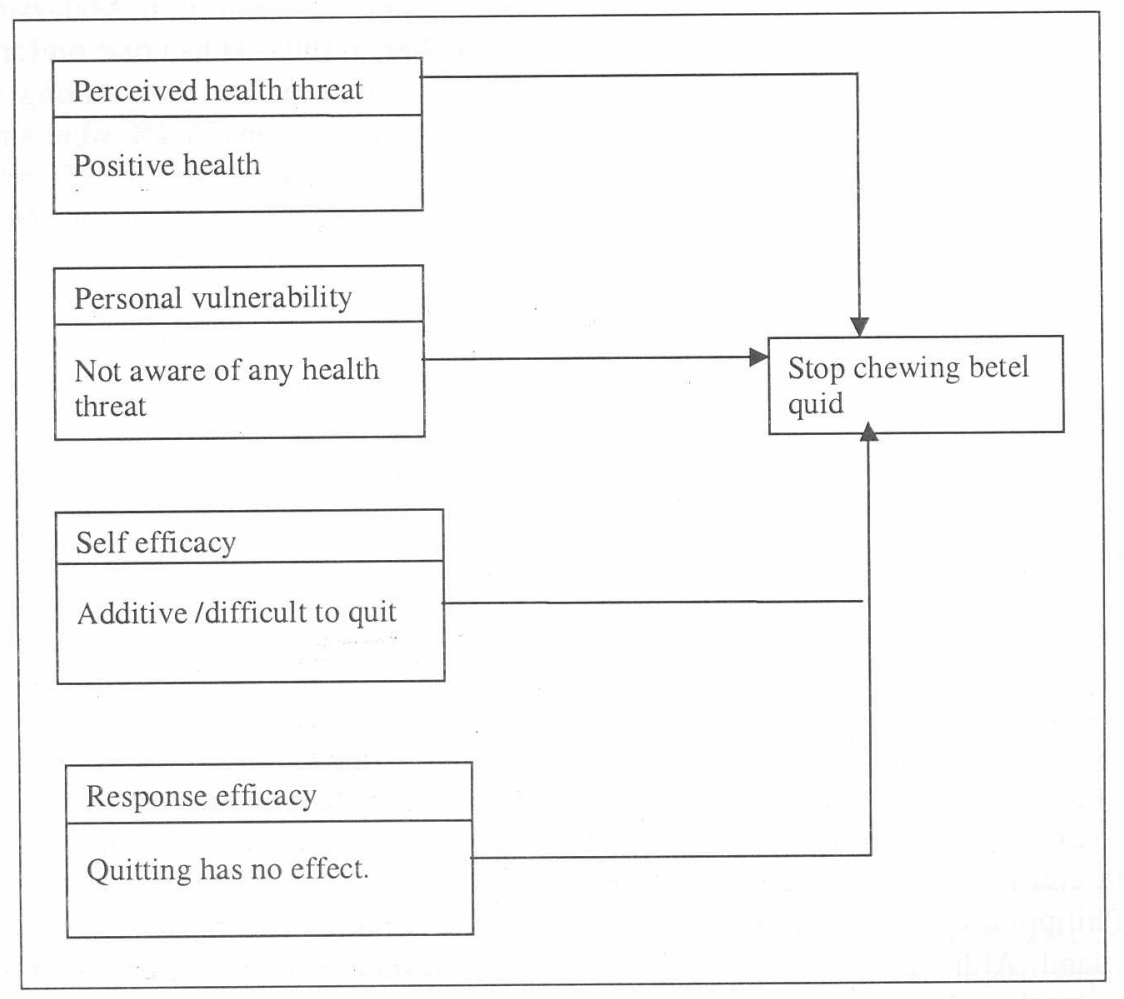

Figure 1 Protection Motivation model for habit of chewing betel quid 
women refugees in San Diego (2). It was found that none of them thought that chewing betel quid is a health risk, although they acknowledged that smoking tobacco causes lung cancer. The same finding was reported among the Bangladeshi population in East London (4). In addition they were not aware of the risk. A study done by Summers (1993) (3) among Bangladeshi women in West Yorkshire reported that $62 \%$ of the sample perceived the practice as "good".

One of the reason people chew betel quid was because they believed that it enhances their health as discussed in the earlier part of this paper. Most of them were unaware of the effect that the habit has on their health especially oral health even though they are exposed to carcinogenic agent (3). Some of them admitted that the practice was highly addictive and they believed that it is difficult to quit (2).

It can be concluded that chewers perceived that this habit gives a more positive health benefit rather than causes detrimental effect on their health, they do not have strong self efficacy in quitting the habit, and they do not think that quitting will have any difference to their life. It seemed that the beliefs on all four components of the model are weak, therefore their beliefs need to be strengthened. Culturally appropriate education and motivation programmes need to be organised in order to provide the facts about the habit and to motivate them to stop practising this habit.

However, it must be aware that the process of changing people's behaviour does not occur overnight. Thus a persistence motivation is needed to ensure that they will be able to change and more importantly to maintain the behaviour. Besides efforts to change this habit by changing their health beliefs, skills to examine the mouth is also recommended in most oral cancer prevention programme literature. By providing this skill to the people, especially those who are being considered as having a high risk to oral cancer would be able to detect early changes in their mouth. Hence the prognosis of early stage of oral cancer will certainly be more promising.

Unlike smoking, betel quid chewing is commonly practised by those in the low socioeconomic status. Among educated people this habit is regarded as disgusting and unacceptable. Thus effort in improving the socioeconomic status of the people will certainly help in curbing this problem.

\section{CONCLUSION}

The habit of chewing betel quid has been practised since ancient times. Although the world has gone through modernization, a significant proportion of people still practises this habit, which to some cultures, is regarded as a disgusting habit. In some cultures it has been integrated into their custom. In some parts of the world, chewing betel quid is a practice of the older generation and in others it has been taken up by the younger generations especially when the products were commercialised.
The betel quid chewing habit has a significant importance to dentistry due to the fact that this habit can cause oral cancer. Substantial evidences show the relationship between the habit and oral cancer. However, it was not perceived as a health threat by the chewers. Therefore it is the responsibility of the dental professional to make them aware of the problem and ultimately change their habit.

\section{REFERENCES}

1) Rooney DF. Betel chewing traditions in South-East Asia. Kuala Lumpur: Oxford University Press. 1993

2) Pickwell SM, Schimelpfening S, Palinkas LA. 'Betelmania' betel quid chewing by Cambodian women in the United States and its potential health effects. Weat J Med 1994; 160:326-330.

3) Summers RM, Williams SA, Curzon MEJ. The use of tobacco and betel quid ('pan') among Bangladeshi women in West Yorkshire. Community Dental Health 1994; 11:12-16.

4) Ahmed S, Rahman A, Hull S. Use of betel quid and cigarettes among Bangladeshi patients in an innercity practice: prevalence and knowledge of health effects. British Journal of General Practice 1997; 47: 431-434.

5) Lu CT, Lan SJ, Hsieh CC, Yang MJ, Ko YC, Tsai CC, Yen YY. Prevalence and characteristics of areca nut chewers among junior high school students in Changhua county, Taiwan. Community Dent Oral Epidemiol 1993; 21: 370-373.

6) Gupta PC. Mouth cancer in India: A new epidemic? Journal of the Indian Medical Association 1999; 97 : 370-373.

7) Zain RB, Ikeda N, Yaacob M. Oral mucosal lesions survey of adults in Malaysia: October 1993-Febuary 1994. Kuala Lumpur 1995.

8) Sankaranarayanan R. Oral cancer in India: An epidemiologic and clinical review. Oral Surg Oral Med Oral Pathol 1990: 69: 325-330.

9) Espie CA, Freedlander E, Campsie LM, Soutar DS, Robertson AG. Psychological distress at follow-up after major surgery for intra-oral cancer. Journal of Psychosomatic Research 1989; 33: 441-448.

10) Gill D. Cancer and precancer in India. Dental Update 1996; May: 149-151.

11) Moore SR, Johnson NW, Pierce AM, Wilson DF. The epidemiology of mouth cancer: A review of global incidence. Oral Diseases 2000; 6: 65-74. 
12) Silverman SJ. Oral Cancer. 4th edition. American Cancer Society 1998.

13) Zain RB, Ikeda N, Gupta PC, Warnakulasuriya KAAS, van Wyk CW, Shrestha P, Axell T. Oral mucosal lesions associated with betel quid - areca nut and tobacco chewing habits: Consensus from a workshop held in Kuala Lumpur, Malaysia. November 25-27 1996. J. Oral Pathol Med 1999; 28: 1-4.

14) Hirayama T. An epidemiologic assessment of oral and oropharyngeal cancer in central and Southeast Asia. Bull WHO 1966; 34: 41-69.

15) Gupta PC, Bhonsle RB, Murti PR, Daftary DK, Mehta FS, Pindborg JJ. An epidemiologic assessment of cancer risk in oral precancerous lesions in India with special reference to nodular leukoplakia. Cancer 1989; 63: 2247-2251.
16) Zain RB, Ikeda N, Razak IA, Axell T, Majid ZA, Gupta PC, Yaacob M. A national epidemiological survey of oral mucosal lesions in Malaysia. Community Dent Oral Epidemiol 1997; 25:377-383.

17) Ali TB, Jalalluddin RL, Abdul Razak I, Zain RB. Prevalence of oral precancerous and cancerous lesions in elderly Malaysians. Asia-Pacific Journal of Public Health 1996-1997; 9: 24-27.

18) Ranadive KJ, Ranadive SN, Shivapurkar NM, Gothuskar SV. Betel quid chewing and oral cancer: Experimental studies on hamsters. International Journal of Cancer 1979; 24: 835-843.

19) Taylor SE. Health Psychology. 2nd edition. New York: McGraw-Hill Inc. 1991. Chapter 4 pp: 76118. 